\title{
First isolation and outbreak of OXA-48-producing Klebsiella pneumoniae in an Irish hospital, March to June 2011
}

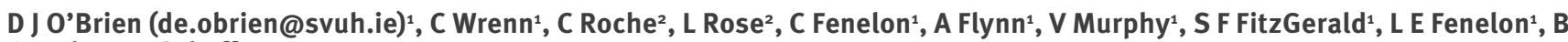
Crowley $^{2}$, K Schaffer ${ }^{1}$

1. Department of Microbiology, St. Vincent's University Hospital, Dublin, Ireland

2. St. James's Hospital, Dublin, Ireland

Citation style for this article:

O’Brien DJ, Wrenn C, Roche C, Rose L, Fenelon C, Flynn A, Murphy V, FitzGerald SF, Fenelon LE, Crowley B, Schaffer K. First isolation and outbreak of OXA-48-producing Klebsiella pneumoniae in an Irish hospital, March to June 2011.

Euro Surveill. 2011;16(29):pii=19921. Available online: http://www.eurosurveillance.org/ViewArticle.aspx?Articleld=19921

Article published on 21 July 2011

Five OXA-48-producing Klebsiella pneumoniae were detected in a tertiary referral hospital in Ireland between March and June 2011. They were found in the clinical isolates of five cases that were inpatients on general surgical wards. None of the cases had received healthcare at a facility outside of Ireland in the previous 12 months. This is the first report of OXA48-producing $K$. pneumoniae in Ireland.

\section{Background}

OXA-48 carbapenemases were first isolated from Enterobacteriaceae in Turkey in 2008 [1]. Since then outbreaks have been described throughout Europe (France, Germany and the United Kingdom [2,3]) and worldwide (Argentina, Lebanon, Israel, Morocco and Tunisia $[2,4,5])$. In November 2010, Ireland was ranked by the European Centre for Disease Prevention and Control (ECDC) as a country with sporadic occurrence of carbapenem-resistant Enterobacteriaceae (CRE) [5]. We describe here the first cases of OXA-48-producing Klebsiella pneumoniae in Ireland.

\section{Case descriptions}

Case 1 was admitted to Surgical ward A for a Whipple's procedure for duodenal adenocarcinoma. Postoperatively he developed an intra-abdominal collection secondary to a hepato-jejunal anastomotic leak. A drain was inserted radiologically, and he was transferred to the intensive care unit (ICU). He was treated with piperacillin/tazobactam and gentamicin and clinically improved. $K$. pneumoniae was grown from intra-abdominal drain fluid taken seven days following drainage of the collection. The isolate was resistant to ertapenem with a minimum inhibitory concentration (MIC) of $8 \mathrm{mg} / \mathrm{L}$ but susceptible to meropenem with a MIC of $0.5 \mathrm{mg} / \mathrm{L}$, and to the third- and fourthgeneration cephalosporins (Table). Modified Hodge test showed carbapenemase production and the isolate was referred to a reference laboratory for molecular evaluation, where it was confirmed by PCR to be an OXA-48 carbapenemase producing $K$. pneumoniae. Regular weekly inpatient screening for OXA-48 carriage was initiated in the ICU and no additional cases were detected in the ICU. The patient initially improved, but died later from complications of a gastrointestinal haemorrhage not related to infection.

Case 2 was admitted to Surgical ward A two days after Case 1. He developed an intra-abdominal collection following reversal of a Hartman's procedure. Two weeks after detection of the first case, $K$. pneumoniae with the same susceptibility pattern as Case 1 was isolated from infected pelvic material. This was subsequently confirmed to be an OXA-48 producer. The patient was treated with tigecycline with successful resolution of the collection. He subsequently developed a bloodstream infection caused by the same isolate and was successfully treated with cefepime.

Case 3 was an inpatient in the same hospital on Surgical ward B and transferred to Surgical ward C during her hospitalisation. She developed a subphrenic collection following colectomy from which $E$. coli (susceptible to carbapenems) was grown. She was treated with drainage of the collection and piperacillin/tazobactam and made a good recovery and antibiotic treatment was discontinued. OXA-48-producing $K$. pneumoniae was subsequently isolated from a midstream urine specimen (Table). She did not have any clinical signs of infection and did not require further antimicrobial treatment.

Case 4 was admitted to Surgical ward A 63 days after Case 1 was discharged and underwent a Whipple's procedure. K. pneumoniae (susceptible to carbapenems) was isolated from biliary drain fluid samples taken pre- and post-operatively. Cases 2 and 3 were still inpatients on Surgical ward $B$ at his time of admission. OXA-48-producing $K$. pneumoniae was subsequently isolated from a midstream specimen of urine taken 23 days post-operatively. There was no clinical evidence of infection and he did not require antimicrobial treatment. 
Case 5 was an inpatient on Surgical ward B. He was initially admitted in April 2011. He had a negative rectal CRE screen at this stage. He was re-admitted for a Whipple's procedure in June 2011. K. pneumoniae susceptible to carbapenems was isolated from a biliary drain removed at operation. He deteriorated acutely five days after the operation following massive pulmonary aspiration of gastric contents and was transferred to the ICU. OXA-48 producing $K$. pneumoniae was isolated from a rectal swab taken to screen for CRE and drain fluid taken at that time. Of note, CRE was not isolated from a rectal screen five days earlier.

\section{Laboratory investigations}

All of the K. pneumoniae isolates had elevated MICs for carbapenem (Table) in automated susceptibility testing (VITEK II, Biomerieux) and in the e-test (Biomerieux) and showed production of carbepenamase enzyme by modified Hodge test. Activity of the carbapenemase was not inhibited by boronic or dipicolinic acid. The presence of OXA-48 carbapenemase in all isolates was confirmed by PCR (as described by Kaczmarek et al. [7]. Comparison of the isolates from cases 1,2 and 3 using Variable Number Tandem Repeat (VNTR) analysis at nine loci showed similarity between the strains, confirming the existence of a clonal outbreak.

\section{Control measures}

After cases 2 and 3 were confirmed to be OXA-48 producers, Surgical wards $A, B$ and $C$ were closed to patient admissions and transfers. Weekly screening for rectal CRE carriage which had previously commenced in the ICU following detection of the first case of CRE was extended to include all patients on the Surgical wards $A, B$ and $C$. In addition, all inpatients that had been on any of the affected wards at the same time as any of the confirmed OXA-48 cases were screened for rectal carriage of CRE. As of 20 July 2011, an additional four cases of OXA-48 rectal carriage had been detected in contacts of confirmed cases.

All CRE-colonised in-patients were placed in single room isolation with contact precautions (long-sleeved gowns and gloves). A programme of enhanced environmental cleaning and disinfection, including disinfection by vapourised hydrogen peroxide, was implemented. Environmental screening of frequently touched surfaces in the ICU and on Surgical wards A, B and C was carried out. Some 150 samples were taken using flocked swabs, which were cultured with a broth enrichment step. CRE was not isolated from any of these samples.

All cases had been in-patients on one of three adjacent surgical wards between which patients are frequently transferred. Most of the ward accommodation consists of six-bedded rooms that share toilet facilities. A detailed epidemiological investigation of the first four cases, including the interventional radiology and theatre departments, did not identify any other link between the cases. Epidemiological investigation is ongoing.

\section{Discussion}

In Ireland, CRE were first reported in 2009; however, these were an isolated case of VIM-producing $K$. pneumoniae [8] and cases of KPC-producing strains [9]. This is the first report of OXA-48 producing $K$. pneumoniae in Ireland. All five cases were patients who had undergone complex abdominal surgery. All had required broad-spectrum antimicrobial treatment (either as antimicrobial prophylaxis for surgery or as treatment for infection) but it is notable that, although carbapenems are used in these wards, none of the cases ever had a

TABLE

Minimum inhibitory concentration profiles of OXA-48 producing Klebsiella pneumoniae, Ireland, March-June 2011 (n=5)

\begin{tabular}{|c|c|c|c|c|c|c|}
\hline Antibiotic & $\begin{array}{c}\text { Case } 1 \\
\mathrm{MIC} \mathrm{mg/L}\end{array}$ & $\begin{array}{c}\text { Case } 2 \\
\mathrm{MIC} \mathrm{mg} / \mathrm{L}\end{array}$ & $\begin{array}{l}\text { Case } 3 \\
\mathrm{MIC} \mathrm{mg} / \mathrm{L}\end{array}$ & $\begin{array}{c}\text { Case } 4 \\
\mathrm{MIC} \mathrm{mg/L}\end{array}$ & $\begin{array}{c}\text { Case } 5 \\
\mathrm{MIC} \mathrm{mg/L}\end{array}$ & $\begin{array}{l}\text { EUCAST } 2011 \text { clinical } \\
\text { breakpoints [6] }\end{array}$ \\
\hline Ertapenem & 8 & 13 & 16 & 1 & 2.0 & $R>1$ \\
\hline Cefuroxime & 4 & 4 & 16 & 4 & $>64$ & $R>8$ \\
\hline Ceftazidime & 1 & 0.5 & 2 & $<1$ & $\ll 1$ & $\mathrm{R}>4$ \\
\hline Cefepime & $<1$ & $<1$ & $\langle 1$ & $<1$ & $<1$ & $R>4$ \\
\hline Aztreonam & 0.5 & $<1$ & $<1$ & $\ll 1$ & $\ll 1$ & $R>4$ \\
\hline Piperacillin/tazobactam & $>64$ & $>64$ & $>64$ & $>64$ & $>64$ & $R>16$ \\
\hline Ciprofloxacin & 18 & 18 & 18 & 1 & 4 & $R>1$ \\
\hline Gentamicin & 132 & 132 & 132 & $>16$ & $>16$ & $R>4$ \\
\hline Tigecycline & 1 & 2 & 8 & $<0.5$ & 8 & $\mathrm{R}>2$ \\
\hline Colistin & 1 & 1 & 1 & $<0.5$ & $<0.5$ & $R>2$ \\
\hline
\end{tabular}

EUCAST: European Committee on Antimicrobial Susceptibility Testing; MIC: Minimum inhibitory concentration. 
history of carbapenem exposure prior to the detection of OXA-48 producing $K$. pneumoniae. None of the OXA48 carrying patients had received healthcare outside Ireland in the previous year. The occurrence of this outbreak of OXA-48 has dramatically changed the epidemiology of CRE in Ireland.

OXA-48 producing Enterobacteriaceae remain difficult to detect as they are often susceptible to third and fourth generation cephalosporins and monobactams, as was the outbreak strain found in our healthcare facility, and therefore vigilance is required. CRE cases have been notifiable in Ireland since March 2011; however, to date, no national compulsory screening programme exists.

As the optimal method of screening for CRE remains to be determined and as CRE may be shed in varying concentrations from the bowel, it is possible that CRE may not be detected in rectal screens of all patients who are colonised. Of note one of the cases reported here had two negative rectal CRE screens but the third screen was subsequently positive. We use the methodology recommended by the United States Centers for Disease Control and Prevention for the detection of CRE in our laboratory [10].

Ongoing rectal screening for CRE carriage continues in all areas where these cases have been found.

\section{Acknowledgements}

The authors would like to acknowledge the assistance of the Health Protection Agency in Colindale UK and the Microbiology Laboratory in St. James's Hospital Dublin Ireland.

\section{References}

1. Carrër A, Poirel L, Eraksoy H, Cagatay AA, Badur S, Nordmann P. Spread of OXA-48-positive-carbapenem-resistant Klebsiella pneumoniae isolates in Istanbul, Turkey. Antimicrob Agents Chemother. 2008;52(8):2950-4.

2. Carrër A, Poirel L, Yilmaz M, Akan OA, Feriha C, Cuzon G, et al. Spread of OXA-48 encoding plasmid in Turkey and beyond. Antimicrob Agents Chemother. 2010;54(3):1369-73.

3. Cuzon G, Naas T, Lesenne A, Benhamou M, Nordmann P. Plasmid-mediated carbapenem-hydrolysing OXA-48 betalactamase in Klebsiella pneumoniae from Tunisia. Int J Antimicrob Agents. 2010;36(1):91-3.

4. Benouda A, Touzani O, Khairallah MT, Araj GF, Matar GM. First detection of an oxacillinase-mediated resistance to carbapenem in Klebsiella pneumonia from Morrocco. Ann Trop Med Parasitol. 2010;104(4):327-30.

5. Grundmann H, Livermore DM, Giske CG, Canton R, Rossolini GM, Campos J, et al. Carbapenem-non-susceptible Enterobacteriaceae in Europe: conclusions from a meeting of national experts. Euro Surveill. 2010;15(46): pii=19711. Available from: http://www.eurosurveillance.org/ViewArticle. aspx?Articleld $=19711$

6. European Committee on Antimicrobial Susceptibility Testing. Breakpoint tables for interpretation of MICs and zone diameters. Version 1.3, January 5, 2011. Available from: http:// www.eucast.org/fileadmin/src/media/PDFs/EUCAST_files/ Disk_test_documents/EUCAST_breakpoints_v1.3_pdf.pdf

7. Prior AR, Roche C, Lynch M, Kelly S, O'Rourke K, Crowley B. First identified case of VIM-producing carbapenem-resistant Klebsiella pneumoniae in the Republic of Ireland associated with fatal outcome. Euro Surveill. 2010;15(50):pii=19752.
Available from: http://www.eurosurveillance.org/ViewArticle. aspx?Articleld $=19752$

8. Roche C, Cotter M, O'Connell N, Crowley B. First identification of Class A carbapenemase-producing Klebsiella pneumoniae in the Republic of Ireland. Eurosurveill. 2009;14(13):pii=19163. Available from: http://www.eurosurveillance.org/ViewArticle. aspx?Articleld=19163

9. Kaczmarek FM, Dib-Hajj F, Shang W, Gootz TD. High-level carbapenem resistance in a Klebsiella pneumoniae clinical isolate is due to the combination of bla(ACT-1) beta-lactamase production, porin OmpK35/36 insertional inactivation, and down-regulation of the phosphate transport porin phoe. Antimicrob Agents Chemother. 2006;50(10):3396-406.

10. Centers for Disease Control and Prevention (CDC). Guidance for control of infections with carbapenem-resistant or carbapenemase-producing Enterobacteriaceae in acute care facilities. MMWR Morb Mortal Wkly Rep. 2009; 58(10):256-60. 